\title{
Role of Cardiac Microstructure Variability on Ventricular Arrhythmogenesis
}

\author{
Dominic G Whittaker, Alan P Benson, Irvin Teh, Jürgen E Schneider, Michael A Colman \\ University of Leeds, Leeds, UK
}

\begin{abstract}
The propagation of cardiac electrical excitation is influenced by tissue microstructure. Reaction-diffusion computational models of cardiac electrophysiology incorporating both dynamic action potential (AP) behaviour and image-based myocardial architecture provide an approach to study the complex organisation of excitation waves within variable myocardial structures.

The role of tissue microstructure (cardiomyocyte and sheetlet orientations) on organ-scale arrhythmic excitations was investigated. Five healthy rat ventricle datasets were obtained using diffusion tensor MRI (DTI). The Fenton-Karma minimal AP model was modified to reproduce the rat AP duration and restitution. Re-entrant scroll waves were initiated in the five anatomical models at ten locations for three microstructure scenarios: (i) isotropic; (ii) anisotropic; and (iii) orthotropic.

Variability in anatomy and microstructure caused simulated scroll waves to self-terminate, remain tachycardia-like, or degenerate into fibrillatory activity. Whilst inclusion of DTI-based microstructure increased total scroll wave filament length to differing extents between the five hearts, overall mean filament dynamics were quantitatively similar under anisotropic and orthotropic conditions. This study highlights the important role of inter-subject structural variability.
\end{abstract}

\section{Introduction}

Cardiac arrhythmias, including ventricular tachycardia (VT) and ventricular fibrillation (VF), are major causes of morbidity and mortality in the developed world, yet remain incompletely understood [1]. As arrhythmias are inherently an organ-scale phenomenon, quantitative characterisation requires visualisation of excitationpropagation throughout the three-dimensional (3D) in vivo beating heart [2]. This is not yet technically feasible, and thus the study of arrhythmias presents a significant challenge. Mapping electrical activity during arrhythmias, using techniques such as depth-resolved optical imaging, is one approach which has been used with some success to shed light on VF mechanisms [3]. Reaction-diffusion models of cardiac electrophysiology, incorporating both dynamic action potential (AP) behaviour and image- based myocardial architecture, offer an alternative way of studying the complex organisation of arrhythmic excitations, which enable direct control of important determinants of arrhythmia dynamics such as ion current properties and tissue structure and anisotropy [4], [5].

The field of computational cardiac modelling is beginning to move beyond the single virtual heart paradigm, as the importance of accounting for intersubject variability is emerging. Recent efforts have focused overwhelmingly on electrophysiological variability, with particular emphasis on how this might affect arrhythmia dynamics and drug response [6]. In this study, we sought to determine to what extent variability in cardiac anatomy and microstructure manifests as differences in arrhythmia dynamics. Quantitative study of this important relationship can improve understanding of the role of myocardial structure in ventricular arrhythmogenesis, as well as shed light on the level of detail required for predictive arrhythmia simulations.

\section{Methods}

\subsection{DTI reconstructions}

Five healthy rat heart reconstructions at $100 \mu \mathrm{m}$ isotropic resolution from ex vivo diffusion tensor MRI (DTI) [7] were used to provide ventricular myocardial structure for reaction-diffusion models of cardiac electrophysiology. The helix and transverse angles and the sheetlet angle, derived from the primary and secondary eigenvectors obtained from DTI, respectively, were computed for each of the ventricular geometries using the coordinate system and method described previously [3]. Variability in characteristic microstructure angles existed between the five data sets (Figure 1).

\subsection{Ventricular tissue simulations}

The Fenton-Karma three variable (FK3V) minimal AP model [8] was modified to reproduce the short $(\sim 50 \mathrm{~ms})$ rat AP duration (APD) and restitution, using experimental data from our lab [9] (Figure 2). Propagation of APs in 3D tissue geometries was described using the monodomain equation, 


$$
\frac{\partial V}{\partial t}=\nabla(\mathrm{D} \nabla V)-\frac{I_{\text {ion }}}{C_{\mathrm{m}}}
$$

where $V$ is the transmembrane voltage, $\mathbf{D}$ is the global conductivity tensor, $I_{\mathrm{ion}}$ is the total ionic current, and $C_{\mathrm{m}}$ is the membrane capacitance. Equation (1) was solved using a finite difference PDE solver based on the explicit forward Euler method and a Strang splitting scheme. The conductivity tensor, $\mathbf{D}$, from Equation (1) is given by

$$
\mathbf{D}=D_{1} \boldsymbol{e}_{1} \boldsymbol{e}_{1}^{\mathrm{T}}+D_{2} \boldsymbol{e}_{2} \boldsymbol{e}_{2}^{\mathrm{T}}+D_{3} \boldsymbol{e}_{3} \boldsymbol{e}_{3}^{\mathrm{T}},
$$

where $D_{1}, D_{2}$, and $D_{3}$ correspond to 'electrical diffusion' in directions along the myocyte orientation axis, perpendicular to myocyte orientation in the sheetlet plane, and normal to the sheetlet plane, respectively, $\boldsymbol{e}_{1}$, $\boldsymbol{e}_{2}$, and $\boldsymbol{e}_{3}$ are the corresponding eigenvectors obtained from DTI, and the superscript $\mathrm{T}$ denotes the vector transpose. $D_{1}$ was set to a value which gave a conduction velocity of $0.6 \mathrm{~m} / \mathrm{s}$ along the myocyte orientation axis [10]. For isotropic simulations, the diffusion coefficients, $D_{\mathrm{i}}$, were set equal to each other, and were scaled (by reducing values radial to the myocyte direction) in the ratios $D_{1}: D_{2}: D_{3}=4: 1: 1$ and $D_{1}: D_{2}: D_{3}=36: 9: 1$ for anisotropic and orthotropic simulations, respectively [2].

A

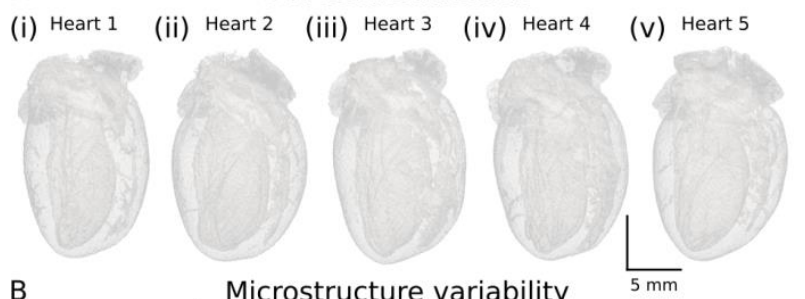

B

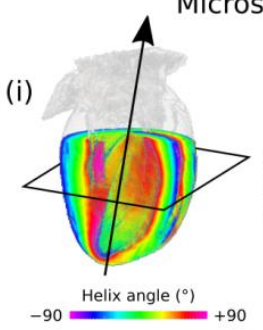

(ii) Helix angle

(iii) Transverse angle
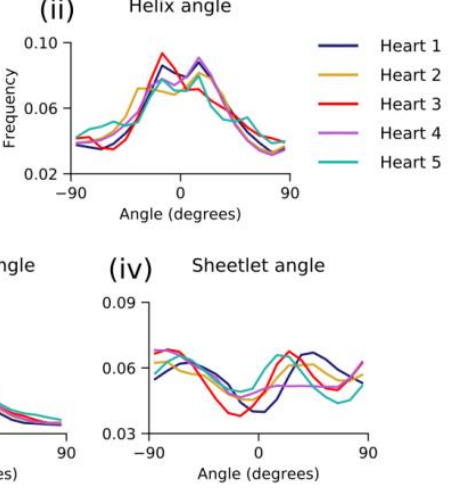

Figure 1: (A) Five healthy whole rat heart reconstructions from ex vivo DTI [7]. (B) (i) Representative cropped ventricular geometry showing helix angle, and variability in (ii) helix angle, (iii) transverse angle, and (iv) sheetlet angle between the five ventricle geometries.
A Rat ventricular cell AP

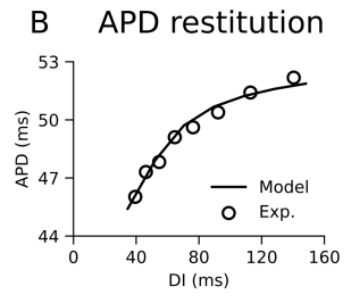

Figure 2: (A) Rat ventricular cell APs from the modified FK3V model at a $5 \mathrm{~Hz}$ pacing rate. (B) Comparison of APD as a function of diastolic interval (DI) from model (solid line) and experiment (points) [9].

For $5000 \mathrm{~ms}$ arrhythmia simulations, conduction velocity was decreased by $50 \%$ to facilitate sustenance of re-entry. The phase distribution method [11] was used to initiate a scroll wave which developed into re-entry at ten different locations in each of the five geometries for three microstructure scenarios (isotropic, anisotropic, and orthotropic). Filaments, which act as 'organising centres' of scroll waves, were tracked by locating phase singularities [12] and counted using a grassfire algorithm. As single filaments can be broken into multiple filaments by the intricate myocardial structure [2], the mean (averaged over the re-entry lifespan) and maximum (the maximum value registered in each simulation) total filament length were chosen as the most useful ways of quantifying wave activity. Values were compared with ANOVA followed by Bonferroni-corrected $t$ tests in R.

\section{Results}

\subsection{Dynamics of a simulated arrhythmia in the five hearts}

Variability in arrhythmia dynamics following initiation of a scroll wave at a prescribed location (left ventricular anterior wall) in each of the five hearts (with orthotropic microstructure) is shown in Figure 3. At time $t=50 \mathrm{~ms}$ following initiation, a single scroll wave drove re-entry in the left ventricular wall, with only minor differences noticeable between the five hearts. By $t=200 \mathrm{~ms}$, greater divergence in scroll wave activity between hearts was observed, and by $t=1000 \mathrm{~ms}$, differences in anatomy and microstructure alone were able to account for whether simulated scroll waves self-terminated, remained as VTlike (single scroll wave filament), or degenerated into VFlike activity (multiple scroll wave filaments).

\subsection{Quantitative analysis of scroll wave filament dynamics}

Simulated arrhythmias were further characterised by quantifying filament dynamics (averaged over $n=10$ simulations for each microstructure scenario within each 
heart), and testing for statistically significant differences in the mean and maximum total filament length. It can be seen that the extent to which both of these measures of the complexity of re-entrant activity were modulated by microstructure differed between the five hearts (Figures 4A,B). In all five hearts inclusion of myocyte orientations (isotropic $\rightarrow$ anisotropic) produced a statistically significant increase in either the mean or maximum total filament length (or both). However, inclusion of sheetlet structure (anisotropic $\rightarrow$ orthotropic) produced an inconsistent effect on the mean value of the mean total filament length (a decrease was observed in Heart 4). Furthermore, no statistically significant differences in the mean total filament length were found between the anisotropic and orthotropic cases, and only a single significant difference was observed for the maximum value. Comparing these measures of filament dynamics between the five hearts (in the anisotropic case) revealed no statistically significant differences (Figure 4C).

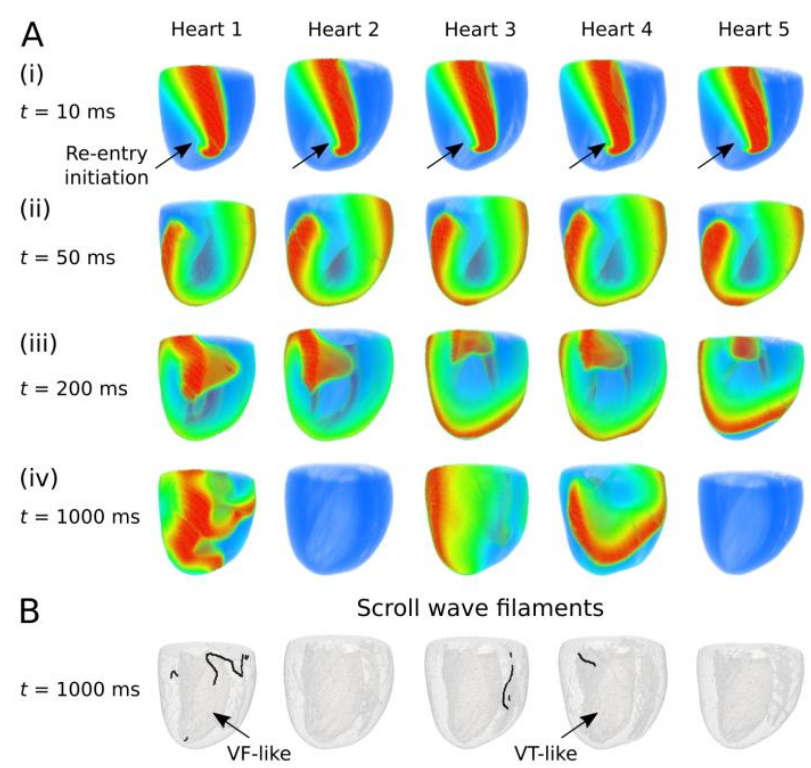

Figure 3: (A) Evolution of a scroll wave initiated on the anterior wall of the left ventricle in each of the five hearts at times (i) $t=10 \mathrm{~ms}$, (ii) $t=50 \mathrm{~ms}$, (iii) $t=200 \mathrm{~ms}$, and (iv) $t=1000 \mathrm{~ms}$. (B) Corresponding scroll wave filaments at $t=1000 \mathrm{~ms}$, which highlight VF- and VT-like activity.

\section{Discussion and conclusions}

In this study, the role of microstructure variability in ventricular arrhythmogenesis was investigated using reaction-diffusion models of cardiac electrophysiology with modified FK3V membrane kinetics and image-based myocardial architecture from high-resolution DTI [7].
It was shown that, in the absence of differences in electrophysiology, anatomy and microstructure alone were able to account for large variation in arrhythmia dynamics. These findings suggest that in structurally normal hearts, it is difficult to predict the lifespan or location of re-entrant waves from specific arrhythmia simulations (in contrast to, for example, post infarction hearts, in which re-entrant circuits tend to anchor to scars [13]). This may have important implications for arrhythmia risk prediction in patients in which the substrate for re-entry is purely electrophysiological (e.g. under gene mutation or pharmacological modulation conditions [14]). In relation to this, cardiac atlases may therefore also have limited predictive ability when it comes to simulating specific arrhythmia protocols. Sample-specific anatomy and microstructure are likely to be necessary for improved predictive arrhythmia simulations for this purpose. Integrating personalised structure with patient-specific electrophysiology is thus likely to be a crucial step towards future translation of computational models of the heart to the clinic [15].

In our simulations, inclusion of myocyte orientations (isotropic $\rightarrow$ anisotropic) was shown to reliably increase the mean and maximum total filament length (averaged over ten simulations). Inclusion of sheetlet organisations (anisotropic $\rightarrow$ orthotropic) produced less consistent effects between the five hearts. Furthermore, no statistically significant differences in mean filament dynamics existed between the anisotropic and orthotropic cases. Nonetheless, our results further suggest that tissue microstructure is a critical determinant of arrhythmia dynamics. Consequently, isotropic tissue simulations may be untenable for quantitative simulations of ventricular arrhythmias, as they significantly underestimate arrhythmia vulnerability. In terms of optimising the level of detail required for predictive arrhythmia simulations, our findings suggest that anisotropic simulations (based on the primary eigenvector from DTI) may be sufficient until greater validation of sheetlet structure exists. When comparing metrics between the five hearts in the anisotropic case (and orthotropic case - not shown), no statistically significant differences were found. This indicates that the general arrhythmia vulnerability (as quantified by the filament dynamics) is not significantly different in similar-sized hearts.

This study shows that ventricular anatomy and microstructure alone can account for large inter-subject variation in arrhythmia dynamics, influencing specific properties such as lifespan and filament length. This further highlights the important role of structural variability in arrhythmogenesis. 
A

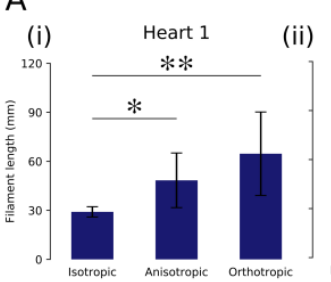

B

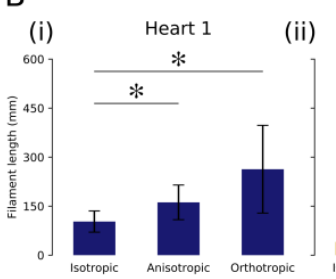

Mean total filament length
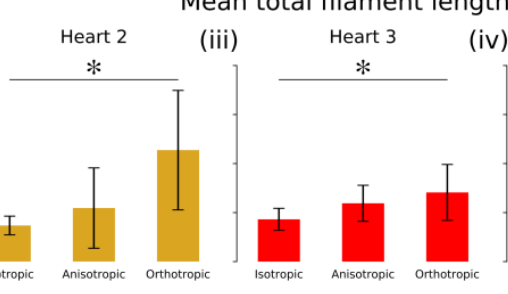

Max. total filament length
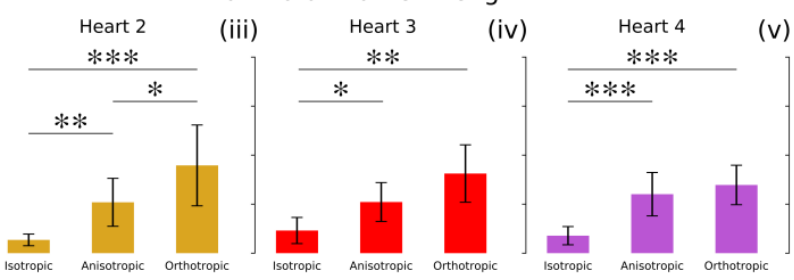
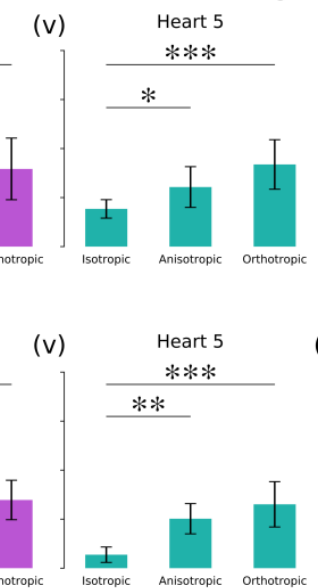

(ii)

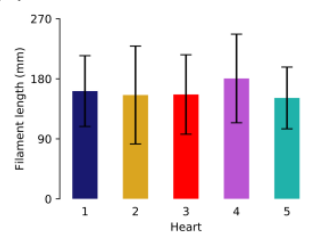

Figure 4: (A) Mean and (B) maximum total filament length in (i) Heart 1, (ii) Heart 2, (iii) Heart 3, (iv) Heart 4, and (v) Heart 5 under isotropic, anisotropic, and orthotropic conditions $(n=10)$. (C) (i) Mean and (ii) maximum total filament length between the five hearts (anisotropic). Results expressed as mean \pm SD. ${ }^{*} p<0.05 ; * * p<0.01 ; * * * p<0.001$.

\section{Acknowledgements}

This work was funded by a Wellcome Trust ISSF Fellowship (204825/Z/16/Z - DGW) and British Heart Foundation project grant (PG/16/74/32374 - APB). All simulations were performed on ARC3 High Performance Computing facilities at the University of Leeds, UK.

\section{References}

[1] J. Jalife, "Ventricular fibrillation: Mechanisms of initiation and maintenance," Annu. Rev. Physiol., vol. 62, no. 1, pp. 25-50, 2000.

[2] A. P. Benson, M. E. Ries, and A. V. Holden, "Effects of geometry and architecture on re-entrant scroll wave dynamics in human virtual ventricular tissues," in Functional Imaging and Modeling of the Heart, 2007, pp. 200-209.

[3] A. P. Benson et al., "Construction and validation of anisotropic and orthotropic ventricular geometries for quantitative predictive cardiac electrophysiology," Interface Focus, vol. 1, no. 1, pp. 101-116, Feb. 2011.

[4] R. H. Clayton et al., "Models of cardiac tissue electrophysiology: Progress, challenges and open questions," Prog. Biophys. Mol. Biol., vol. 104, no. 1-3, pp. 22-48, Jan. 2011.

[5] M. J. Bishop and G. Plank, "The role of fine-scale anatomical structure in the dynamics of reentry in computational models of the rabbit ventricles," J. Physiol., vol. 590, no. 18, pp. 4515-4535, 2012.

[6] H. Ni, S. Morotti, and E. Grandi, "A heart for diversity: Simulating variability in cardiac arrhythmia research," Front. Physiol., vol. 9, Jul. 2018.

[7] I. Teh et al., "Resolving fine cardiac structures in rats with high-resolution diffusion tensor imaging," Sci. Rep., vol. 6, p. 30573, Jul. 2016.

[8] F. Fenton and A. Karma, "Vortex dynamics in threedimensional continuous myocardium with fiber rotation:
Filament instability and fibrillation," Chaos Interdiscip. $J$. Nonlinear Sci., vol. 8, no. 1, pp. 20-47, Mar. 1998.

[9] D. Benoist et al., "Systems approach to the study of stretch and arrhythmias in right ventricular failure induced in rats by monocrotaline," Prog. Biophys. Mol. Biol., vol. 115, no. 2-3, pp. 162-172, Aug. 2014.

[10] R. D. Walton, A. P. Benson, M. E. L. Hardy, E. White, and O. Bernus, "Electrophysiological and structural determinants of electrotonic modulation of repolarization by the activation sequence," Front. Physiol., vol. 4, Oct. 2013.

[11] V. N. Biktashev and A. V. Holden, "Reentrant waves and their elimination in a model of mammalian ventricular tissue," Chaos Interdiscip. J. Nonlinear Sci., vol. 8, no. 1, pp. 48-56, Mar. 1998.

[12] M.-A. Bray and J. P. Wikswo, "Use of topological charge to determine filament location and dynamics in a numerical model of scroll wave activity," IEEE Trans. Biomed. Eng., vol. 49, no. 10, pp. 1086-1093, Oct. 2002.

[13] H. J. Arevalo et al., "Arrhythmia risk stratification of patients after myocardial infarction using personalized heart models," Nat. Commun., vol. 7, p. 11437, May 2016.

[14] D. G. Whittaker, H. Ni, A. P. Benson, J. C. Hancox, and H. Zhang, "Computational analysis of the mode of action of disopyramide and quinidine on hERG-linked short QT syndrome in human ventricles," Front. Physiol., vol. 8, Oct. 2017.

[15] R. A. Gray and P. Pathmanathan, "Patient-specific cardiovascular computational modeling: Diversity of personalization and challenges," J Cardiovasc. Transl. Res., vol. 11, no. 2, pp. 80-88, Apr. 2018.

Address for correspondence.

Dominic G Whittaker.

Garstang 7.53,

University of Leeds,

Leeds, UK,

LS2 9JT,

d.whittaker@leeds.ac.uk 